\title{
Trabalho industrial e câncer de pulmão*
}

\author{
Industrial work and lung cancer
}

\author{
Victor Wünsch Filho, Cecilia Magaldi, Neusa Nakao, José E.C. Moncau \\ Núcleo de Investigação em Trabalho e Saúde. Instituto de Saúde/SES-SP - Brasil (V.W.F.) \\ Departamento de Epidemiologia da Faculdade de Saúde Pública da Universidade de \\ São Paulo - Brasil (V.W.F., N.N.) \\ Faculdade de Medicina de Botucatu/UNESP - Brasil (C.M.) \\ Departamento de Medicina Preventiva da Escola Paulista de Medicina - Brasil (J.E.C.M.)
}

\begin{abstract}
Com base em populaçōes hospitalares, foram entreviștados 316 casos e 536 controles para obtençăo de informaçōes sobre histórias de trabalho, tabagismo, tabagismo passivo, ocorréncia de outros cánceres na familia, migraçăo e situaç̧̌xo socioeconơmica. Os entrevistadores utillzaram um questionário padronizado. Casos $\theta$ controles foram emparelhados por hospital, sexo $\theta$ idade. O estudo foi desenvoivido entre $1^{2}$ de julho de 1990 e 31 de janeiro de 1991 em 14 hospitais da Regiáo Metropolitana de São Paulo, SP (Brasil). Foi elaborada uma classificação por escores como forma de ordenar os Indivíduos estudados segundo suas histórias de exposiçōes ocupacionais a cancerígencs, conhecidos para o pulmāo, acumulando informaçōes sobre a exposição nos locais de trabalho durante a vida, considerando o tipo de atividade, setor de trabalno e tempo em cada emprego. A análise de regressăo logistica nåocondictonal mostrou odds ratio: $1,97(95 \%$ iC: 1,52 a 2,55$)$ para os trabalihadores com maiores escores de exposiçäo. Os resultados permitiram inferir que trabalhadores ligados aos setores de producāo de diferentes ramos industriais têm duas vezes o risco de desenvolverem neoplasia pulmonar quando comparados aos trabalhadores envolvidos em atividades näo-industriais.
\end{abstract}

Neoplasias pulmonares, epidemiologia. Exposição ocupacional, efeitos adversos. Indústrias.

\section{Introduçảo}

O câncer tem distribuição mundial, sendo uma importante causa de 6 bito entre indivíduos na idade produtiva. Para a populaçāo brasileira, acima de 40 anos de idade, representa a segunda causa de morte, situando-se atrás apenas das doenças cardiovasculares. As neoplasias malignas da traquéia, brônquios e pulmōes que, na prática, nāo ocorrem na faixa etária de 20 anos, contribuíram com $17 \%$ das mortes por câncer na população masculina brasileira em 1988, situando-se como a primeira causa de morte por câncer nos homens de todas as idades ${ }^{7,16,17}$.

A neoplasia pulmonar é a principal incidência de câncer em muitos países, particulamente na
Europa Ocidental e na América do Norte, onde a taxa de incidência anual excede a 100 por 100.000 em algumas populaçōes masculinas. Na China, India e vários países latino-americanos e africanos a taxa de incidência anual năo excede 25 por 100.000 habitantes ${ }^{20}$.

A tendência do câncer de pulmão no Brasil, com a maior incidência ocorrendo nos Estados do Sul e Sudeste, mostra paralelo com outros países, indicando ser esta uma doença mais comum em áreas urbanas e industrializadas. Na cidade de São Paulo, o câncer de pulmão corresponde a terceira maior ocorrência entre todos os cânceres, com inciđência anual de 36,5 por 100.000 habitantes ${ }^{18,21}$.

Nos Estados Unidos, a neoplasia pulmonar, embora represente $15 \%$ da morbidade por câncer,

\footnotetext{
"Parte do projeto "Riscos ocupacionais e câncer de pulmāo na Região Metropolitana de Säo Paulo" financiado pelo Convênio Programo Metropolitano de Saúde/Banco Mundial e desenvolvido no Instituto de Saúde/SES-SP.

Separatas/Reprints: Cectlia Mogaldi - Rua Quintino Bocaiuva, I40I - 18607-720 - Botucatu, SP - Brasil

Ediçāo subvencionada pela FAPESP. Processo 95/2290-6.

Recebido em 10.10.1994. Aprovado em 10.04.1995.
} 
contribui com um quarto das mortes por neoplasias malignas. Apesar das significativas taxas de morbidade, mortalidade e, particularmente, letalidade, o carcinoma broncogênico é uma doença potencialmente prevenível, uma vez que tanto os fatores ligados ao estilo de vida, a exemplo do tabagismo, como fatores ambientais, particularmente as exposições industriais, têm sido identificados como exercendo um importante papel na etjologia da doença ${ }^{3,20}$.

O nível de exposição a substâncias cancerígenas nos locais de trabalho varia de país para país e, em determinado país, de região para região. Estas variações dependem do nível de desenvolvimento, do grau de modernização e de incorporação de tecnologia nos processos industriais e, ainda, da vigilância exercida nos ambientes de trabalho.

Do ponto de vista das ações de saúde pública, é relevante determinar os fatores ocupacionais vinculados ao câncer, de forma a removê-los ou controlá-los. E mais fácil intervir sobre a exposição ocupacional do que sobre fatores relacionados a hábitos pessoais, fortemente influenciados pela cultura da sociedades.

A quase totalidade dos estudos sobre ocupação e câncer teve como base populações trabalhadoras de países capitalistas desenvolvidos, cujos processos de industrialização são mais antigos e estruturados. $O$ objetivo do presente estudo é analisar a associação entre ocupação e neoplasia pulmonar no contexto de um país subdesenvolvido e recentemente industrializado. Como hipótese de trabalho, considerou-se que se o câncer tem distribuição distinta entre os sexos, entre indivíduos de diferentes faixas etárias e entre grupos de fumantes e nāo-fumantes, também deveria apresentar diferenças de ocorrência entre indivíduos exercendo diferentes tipos de trabalho e, ainda, entre populações vivendo e trabalhando em sociedades industrializadas com características socioculturais diversas. Nesse sentido, o estudo busca avaliar os riscos ocupacionais para a neoplasia pulmonar na população da Regiăo Metropolitana de São Paulo (RMSP).

\section{Populaçäo e Método}

Este é um estudo de caso-controle com base em populaçōes hospitalares. Os dados referem-se a 316 casos de câncer de pulmão e 536 controles com outras doenças que nāo câncer. Os pacientes foram entrevistados no período de $1^{\circ}$ de julho de 1990 a 31 de janeiro de 1991 , em 14 hospitais que concentram o atendimento a pacientes com câncer de pulmão na RMSP.

\section{Coleta de Dados}

Os casos e controles selecionados para o estudo foram entrevistados através de um questionário padronizado composto por 93 questōes, e sua aplicação exigiu um tempo médio de $30 \mathrm{~min}$ por um entrevistador treinado*. O questionário foi estruturado para se obter informaç̄es sobre migraçāo, outros cânceres na famf́lia, tabagismo, tabagismo passivo (na infância e na vida adulta), história clínica e história ocupacional (para cada emprego referido, com permanência maior que seis meses, foi aplicado um questionário específico para se obter informações sobre a atividade da empresa, setor e tempo de trabalho, exposição a agentes físi$\cos$ e substâncias químicas e, ainda, tabagismo passivo no local de trabalho).

Os questionários foram codificados e digitados duas vezes. Posteriormente, as digitações foram comparadas para detectar possíveis erros. A estrutura do banco de dados foi feita no programa $D$ Base III Plus.

\section{Seleção dos Casos}

Os casos foram identificados nos hospitais selecionados da RMSP, no período compreendido entre $1^{\circ}$ de julho a 31 de dezembro de 1990 . Todos os pacientes com diagnóstico ou suspeita diagnóstica de neoplasia pulmonar atendidos nesses serviços eram potenciais participantes do estudo. Os que referiram ter vindo para São Paulo apenas para diagnóstico e/ou tratamento foram excluídos. Os participantes deveriam residir na RMSP há seis ou mais meses antes da data da entrevista.

Embora fossem entrevistados pacientes com suspeita da doença, a definição diagnóstica faziase pelo exame do prontuário do paciente. Os pacientes que não tiveram confirmada a suspeita de carcinoma broncogênico e sim outro diagnóstico clínico que nāo câncer, foram reclassifícados como controles. Os demais, com outros cânceres, foram excluídos do estudo.

\section{Definição da Doença}

Os critérios diagnósticos foram considerados em uma ordem hierárquica: histologia, citologia,

* Copias do questionario estāo disponiveis com os autores. 
broncoscopia, diagnóstico por imagem (radiografia de tórax, tomografia computadorizada, etc.), cirurgia, necrópsia, testes laboratoriais e exame clínico. Entảo, se um paciente teve o diagnóstico de câncer de pulmão confirmado pelo exame histológico, não foi necessário recorrer ao resultado de outros exames. $\mathrm{Na}$ ausência de exames histológicos ou citológicos positivos, o diagnóstico firmado pela broncoscopia ou tomografia foi aceito. Entretanto, os dados clínicos sempre foram considerados na conclusão do diagnóstico. Dưvidas eventuais relativas ao diagnóstico foram esclarecidas com o clínico responsável pelo paciente.

\section{Seleção dos Controles}

Com o propósito de atingir, pelo menos, a razão de 1:1 entre casos e controles, para cada caso buscou-se entrevistar três controles, emparelhados por hospital, idade (diferença máxima de três anos) e sexo. Os controles foram escolhidos entre pacientes internados ou em tratamento ambulatorial, por qualquer outra doença que não câncer, nos mesmos hospitais onde foram detectados os casos. Os controles foram, preferencialmente, procurados dentro do mesmo hospital onde o caso foi identificado. Entretanto, como alguns dos hospitais selecionados atendem apenas pacientes com câncer, foi necessário encontrar alternativas para a busca de controles, porém, sempre dentro das instituições que fazem parte do estudo. Os pacientescontroles foram entrevistados entre $1^{\circ}$ de julho de 1990 e 31 de janeiro de 1991.

Os controles tiveram seus diagnosticos definidos através da análise dos prontuários hospitalares. Pacientes entrevistados como controles, mas que tiveram diagnóstico firmado de neoplasia pulmonar, foram reclassificados como casos.

\section{Definiçãa da Exposição Ocupacional}

Exposiçâo neste estudo significa exposiçāo potencial a agentes cancerígenos para o câncer de pulmão. A avaliaçăo da exposição foi feita através da história ocupacional individual. Para cada emprego ao qual o paciente, caso ou controle, esteve vinculado por pelo menos seis meses foram levantadas informaçōes detalhadas em relação a cinco variáveis principais:

Setor da economia: Refere-se à inserção da empresa por setor econômico, por exemplo, agropecuário, comércio, indústria, serviços, entre outros.
Ramo de atividade: Esta variável caracteriza a empresa. Exemplos: bancos; comércio atacadista ou varejista; construção civil; indústrias como a química ou a metalúrgica. Para codificar esta variável foi usada a classificação do INSS (Instituto Nacional da Seguridade Social) e da FUNDACENTRO (Fundação Jorge Duprat Figueiredo de Segurança e Medicina do Trabalho).

Setor de trabalho: Variável que remete à atividade especifica que a pessoa desenvolvia na empresa. Esta informaçăo permitiu a melhor codificação da variável ramo de atividade e também saber se a atividade desenvolvida pelo trabalhador estava proxima a uma fonte de exposiçăo cancerigena.

Referência a potenciais substâncias cancerigenas no trabalho: $O$ exame desta variável permitiu avaliar, no ambiente de trabalho, a presença de substâncias que poderiam ser deletérias para a saúde, especificamente substâncias cancerígenas. Para codificação desta variável utilizou-se a listagem de susbstâncias e agentes cancerigenos da Organização Internacional do Trabalho (OIT) ${ }^{8}$.

Tempo de trabalho: Variável temporal da exposição ocupacional em cada emprego.

A partir dessas variáveis foi desenvolvida uma classificação por escores que se denominou "Classificação de Exposição Ocupacional a Cancerígenos por Escores".

Os ramos de atividade foram classificados em uma escala de 0 a 10 . $O$ escore 0 (zero) foi atriburdo a pessoas que informaram nunca terem trabathado fora de casa. $O$ escore 10 foi atribuído a indivíduos expostos a ramos industriais específicos: artefatos de borracha e pneumáticos; derivados do petróleo e hulha; plásticos; produtos minerais não metálicos; metalurgia, mecânica e material elétrico e eletrônico. Portanto, por hipotese, as pessoas envolvidas em um ramo de atividade ao qual se atribuiu escore 10 teriam maior risco de exposição a substâncias cancerígenas do que as classificadas no escore 0 . Entre o escore 0 e o 10 há uma gradaçāo crescente no sentido do menor ao maior potencial de exposição a substâncias cancerígenas, considerando-se as características do processo de produção e estimativas da concentração destas substâncias nos locais de trabalho.

Para o setor de trabalho a escala variou de 1 a 5. Na atribuição do escore considerou-se a combinação de duas variáveis: (a) segundo critério dos pesquisadores, os participantes do estudo foram classificados como potencialmente expostos (trabalho em setores ligados diretamente à produção) 
$\mathrm{e}$, potencialmente não-expostos (trabalho nos demais setores); e (b) informação dos pacientes sobre a exposiçăo a substâncias químicas no local de trabalho. Estas substâncias foram categorizadas como gerais ou cancerigenas a partir de classificação da $\mathrm{OTT}^{3}$.

Da combinação das variáveis ramo de atividade e setor, o escore de cada trabalho exercido poderia variar entre 0 e 50 . $O$ tempo entrou na composiçāo do escore como variável contínua. Portanto, dado que um indivíduo tivesse trabalhado em determinado ramo de atividade com escore 5 , no setor de trabalho com escore 2, o produto destes dois escores definiria um escore 10 . Tendo o entrevistado trabalhado nesse emprego, por 20 anos, o escore seria 200 . Na situação de ter sido o único vínculo empregatício da pessoa na vida, 200 seria o seu escore individual final. Tendo havido outros empregos, os escores dos diferentes empregos seriam somados, resultando o valor final. Portanto, cada paciente integrante do estudo, caso ou controle, teve atribuído um escore individual para a exposição ocupacional.

$O$ objetivo da construção desta classificação não foi mensurar a quantidade da exposição, mas ordenar os indivíduos segundo a potencial exposiçāo ocupacional cumulativa a cancerígenos.

\section{Outras Variáveis Avaliadas}

Além da exposição ocupacional, outros fatores com possível efeito na ocorrência de neoplasia pulmonar foram avaliados: tabagismo, tabagismo passivo, migração, outros cânceres na família e estrato socioeconômico. $O$ objetivo do levantamento destas variáveis era controlá-las na análise.

Tabagismo: Para cada paciente tabagista (definido no estudo como quem referiu ter fumado 50 ou mais cigarros na vida) foi feita uma história detalhada incluindo idade do início do tabagismo, variações do tabagismo na vida, tipo de tabaco usado, quantidade média de cigarros fumados e intensidade da inalaçăo.

Tabagismo passivo: Foram obtidas informações sobre a exposição passiva ao tabagismo na infância e na vida adulta do paciente (domicilio e trabalho). Explorou-se a intensidade e o tempo desta exposição passiva ao tabaco.

Migraçāo: A história migratória dos entrevistados permitiu estudar as variaçōes da ocorrência da neoplasia pulmonar em relação à maior ou menor exposiçảo ao meio urbano ou rural.
Outros canceres na familia: Rastreou-se a ocorrência de outros cânceres entre parentes consangüíneos do entrevistado.

Estrato socioeconômico: Esta variável foi avaliada através de informações sobre o índice de aglomeração no domićlilio, escolaridade, categoria profissional e renda familiar per capita.

\section{Métodos de Análise dos Dados}

Apos a análise descritiva dos dados, examinouse o efeito isolado das variáveis trabalho industrial, tabagismo, tabagismo passivo, outros cânceres na familia, história migratória e estrato socioeconômico na ocorrência da neoplasia pulmonar. Foi estimado o odds ratio (OR)* e o respectivo intervalo de confiança (IC) em núvel de $95 \%$.

Nas variáveis que mostraram associaçāo importante com a neoplasia pulmonar investigouse a tendência dose-resposta. Estas variáveis foram tratadas como de confusāo e, juntamente com idade e sexo, controladas pela técnica de estratificação proposta por Mantel-Haenszel ${ }^{14}$.

Como última etapa da análise, para examinar o efeito conjunto da exposiçăo aos diferentes fatores, o índice de exposiçảo ocupacional cumulativa de cada integrante do estudo foi analisado simultaneamente com tabagismo, outros cânceres na familia, sexo $e$ idade, através da regressāo logística nāo-condicional ${ }^{2}$, portanto, não considerando o emparelhamento.

Nas análises estratificadas e de regressão logística, para observação do efeito de exposição ocupacional, os escores dos casos e controles (que apresentaram variação de zero a 1.732) foram distribuídos em quatro aglomerados, definidos pelas diferenças entre as médias dos grupos, segundo o método de áglomerados hierárquicos ${ }^{\imath}$, a partir dos valores dos escores na escala logarítmica.

Para os procedimentos de análise foram utilizados dois programas estatístico-epidemiologicos: SAS e EGRET.

\section{Resultados}

O conjunto de dados do estudo é formado por 852 observaçóes, 316 casos e 536 controles, o que define a razảo de 1:1, 7 entre casos e controles.

* Em portugués nāo há nomenclatura epidemiológica padronizada e muitos termos são repraduzidos conservando, $\mathrm{em}$ geral, a terminologia original em inglês. $O$ termo odds ratio designa a medida de associaça utilizada nos estudos de caso-controle ${ }^{r 2}$. 


\section{Análise Descritiva}

Entre as 852 pessoas do estudo registraram-se 331 diferentes ocupaçōes em 3.016 empregos. A rotatividade foi, em média, de 3,5 empregos, sendo discretamente maior nos casos (média 3,8 ) do que entre os controles (média 3,4). Os homens apresentaram rotatividade maior (média de 4,2 empregos) do que as mulheres (média de 2,2 empregos). $\mathrm{O}$ tempo médio de trabalho registrado foi de 40,7 anos para os homens e 26,1 para as mulheres.

Algumas características básicas de casos e controles sāo mostradas na Tabela 1 .

Tabela 1 - Características dos casos e controles.

\begin{tabular}{|c|c|c|c|c|}
\hline \multirow{2}{*}{$\begin{array}{l}\text { Características da } \\
\text { populaçāo }\end{array}$} & \multicolumn{2}{|c|}{ Casos } & \multicolumn{2}{|c|}{ Controles } \\
\hline & $\mathrm{N}^{8}$ & $\%$ & $N^{2}$ & $\%$ \\
\hline \multicolumn{5}{|l|}{ idade (anos) } \\
\hline$<40$ & 3 & $\begin{array}{l}1,0 \\
82\end{array}$ & 12 & $\begin{array}{r}2,2 \\
121\end{array}$ \\
\hline 40 a 49 & 26 & $\begin{array}{r}8,2 \\
3+0\end{array}$ & $\begin{array}{r}65 \\
168\end{array}$ & $\begin{array}{l}12,1 \\
31,4\end{array}$ \\
\hline 50 a 50 & 98 & & 168 & 31,4 \\
\hline 60 a 69 & 111 & 35,1 & 193 & 36.0 \\
\hline 70 e mais & 78 & 24,7 & 98 & 18,3 \\
\hline \multicolumn{5}{|l|}{ Sexo } \\
\hline Masculino & 236 & 74,7 & 334 & 62,3 \\
\hline Feminino & 80 & 25,3 & 202 & 37,7 \\
\hline \multicolumn{5}{|l|}{ Tipo histologico do tumor } \\
\hline Epidermóide & 135 & 42,7 & & \\
\hline Adenocarcinoma & 79 & 25,0 & & \\
\hline Pequenas cólulas & 49 & 15,5 & & \\
\hline Grandes células & 15 & 4,8 & & \\
\hline Indiferenciado & 38 & 12,0 & & \\
\hline \multicolumn{5}{|l|}{ Estrato socioeconoimico } \\
\hline Nivel baixo" & 95 & 30,1 & 187 & 34,9 \\
\hline Diferente do nivel baixo & 221 & 69,9 & 349 & 65,1 \\
\hline \multicolumn{5}{|c|}{$\begin{array}{l}\text { O paciente fol classificado no estrato socioeconómico } \\
\text { de nivel baixo quando pelo menos duas das } \\
\text { seguintes condiçōes estavam presentes: (a) quatro } \\
\text { ou mais pessoas por dormitório no domícilio; (b) anal- } \\
\text { fabeto ou primário incompleto; (c) categoria profis- } \\
\text { sional do paciente classificada como pertencente à } \\
\text { ciasse operária. }\end{array}$} \\
\hline
\end{tabular}

Embora, na faixa etária com maior número de observaçōes (50 a 69 anos), note-se equilíbrio entre os casos e controles, no conjunto, o grupo dos controles é formado por uma populaçâo mais jovem. Com relação ao gênero, há mais mulheres entre os controles.

Portanto, apesar de, pela logística do estudo, serem rastreados três controles por caso, com critérios definidos de emparelhamento, os dados indicam que foi mais fácil encontrar e entrevistar pacientes do sexo feminino de faixas etárias mais jovens.
Em 98\% dos casos o diagnóstico de neoplasia pulmonar foi feito por biópsia tissular ou por citologia oncótica. Nos restantes (seis casos) o diagnóstico foi definido por broncoscopia e/ou por imagem. Estes seis casos entraram no estudo como carcinoma broncogênico de tipo histológico indiferenciado.

Os 536 controles entrevistados tiveram uma grande variedade đe diagnósticos hospitalares. A pneumologia e cardiologia foram as especialidades que mais fomeceram controles $(55,0 \%)$. $O$ diagnóstico mais comum foi a tuberculose pulmonar (14\%), seguido das doenças pulmonares obstrutivas crônicas ( $8 \%)$, da hipertensão arterial sistêmica $(6 \%)$ e da insuficiência cardíaca congestiva $(5,6 \%)$. Os demais controles tiveram diagnósticos variados: infarto agudo do miocárdio, pneumonias, diabetes mellitus, fraturas, artrite reumatóide, síndrome da imunodeficiência adquirida (AIDS).

\section{Análise do Efeito Isolado das Variáveis}

O efeito isolado dos fatores de risco considerados neste estudo para a neoplasia pulmonar são mostrados na Tabela 2.

$O$ tabagismo surge neste primeiro plano de análise como o fator etiológico mais intensamente associado à neoplasia pulmonar, OR: 4,15 (95\% IC: 2,79 a 6,20 ), embora o critério para a classificação dos tabagistas nesta fase tenha sido amplo e inespecífico (ter fumado 50 ou mais cigarros durante toda a vida). A presença de câncer de pulmão en familiares mostrou um OR também alto: 3,20 , embora nāo significativo estatisticamente (95\% IC: 0,97 a 11,08). A história de dez ou mais anos de trabalho industrial revelou OR: 1,24 e não significativo (95\% IC: 0,85 a 1,80$)$. As demais variáveis não mostraram efeitos estatisticamente significantes.

O consumo de cigarros é geralmente quantificado nos estudos epidemiológicos considerando-se o produto do número de maços fumados por dia pelo número de anos de tabagismo (maços/ano). A tendência de dose-resposta do tabagismo para o câncer de pulmão foi analisada para os padrões compostos por tabagistas, ex-tabagistas e nãotabagistas. O grupo dos tabagistas compunha-se daqueles indivíduos que referiram estar fumando regularmente na época da entrevista e, também, por aqueles que não estavam fumando mas que haviam interrompido o hábito de fumar há menos de 1 ano da data da entrevista. O grupo de ex-tabagistas constituía-se daqueles que não fumavam na época em que foram entrevistados e que haviam aban- 
Tabela 2 - Distribuiçāo de casos e controles, segundo os principais fatores de risco estudados e odds ratio (OR) correspondente.

\begin{tabular}{|c|c|c|c|c|c|c|}
\hline \multirow[t]{2}{*}{ Variáveis } & \multicolumn{2}{|c|}{ Casos } & \multicolumn{2}{|c|}{ Controles } & \multirow[t]{2}{*}{ OA } & \multirow{2}{*}{$\begin{array}{l}\text { Intervalo } \\
\text { confiança } \\
95 \% \%^{*}\end{array}$} \\
\hline & $N^{2}$ & $\%$ & $N^{2}$ & $\%$ & & \\
\hline \multicolumn{7}{|l|}{$\begin{array}{l}\text { Trabalho industrial } \\
\text { Trabalho industrial de qualquer ramo em } \\
\text { qualquer periodo da vida por pelo menos }\end{array}$} \\
\hline & 62 & 19,6 & 88 & 16,4 & 1,24 & $0,85-1,80$ \\
\hline \multicolumn{7}{|l|}{ Tabagismo } \\
\hline toda a vida & 278 & 87,9 & 342 & 63,9 & 4,15 & $2,79 \cdot 6,20$ \\
\hline \multicolumn{7}{|l|}{ Tabagismo Passivo } \\
\hline Moradia na infância (intenso) & 55 & 17,4 & 77 & 14,4 & 0,96 & $0,64-1,47$ \\
\hline $\begin{array}{l}\text { Vida adulta (exposiçāo na moradia por } \\
\text { mais de } 20 \text { horas semanais por mais de }\end{array}$ & & & & & & \\
\hline 5 anos) & 47 & 14,9 & 122 & 22,8 & 0,52 & $0,35-0,78$ \\
\hline \multicolumn{7}{|l|}{ Câncer na tam/lia } \\
\hline Câncer de pulmaxo & 9 & 2,8 & 5 & 0,9 & 3,20 & $0,97-11,08$ \\
\hline Qualquer câncer & 56 & 17,7 & 75 & 14,0 & 1,19 & $0,78-1,81$ \\
\hline \multicolumn{7}{|l|}{ Historia migratoria } \\
\hline $\begin{array}{l}\text { Ter nascido e sempre residido ne cidade } \\
\text { de São Paulo }\end{array}$ & 37 & 11,7 & 63 & 11,8 & 1,10 & $0,69-1,73$ \\
\hline \multicolumn{7}{|l|}{ Estrato socioeconómico } \\
\hline Nivel Baixo** & 95 & 30,1 & 187 & 36,2 & 0,80 & $0,59-1,10$ \\
\hline
\end{tabular}

donado o hábito de fumar há 1 ou mais anos. Os não-tabagistas caracterizavam-se por nunca terem

Tabela 3 - Distribuiçāo de casos e controles, segundo a variável tabagismo, categorizada em nāo-tabagistas, ex-tabagistas e tabagistas e adds ratio (OR) correspondente.

\begin{tabular}{|c|c|c|c|c|c|c|}
\hline \multirow[t]{2}{*}{ Variáveis } & \multicolumn{2}{|c|}{ Casos } & \multicolumn{2}{|c|}{ Controles } & \multirow[t]{2}{*}{ OF } & \multirow{2}{*}{$\begin{array}{c}\text { Intervalo } \\
\text { contilança } \\
95 \% \%^{\circ}\end{array}$} \\
\hline & $\mathrm{N}^{\mathrm{a}}$ & $\%$ & $\mathrm{~N}^{2}$ & $\%$ & & \\
\hline dbagistas"* & 38 & 12,0 & 194 & \multicolumn{2}{|c|}{$36,21,00^{* * *}$} & \\
\hline Ex-tabagistas"*** & 99 & 31,3 & 152 & \multicolumn{2}{|c|}{$28,43,33$} & $2,12-5,23$ \\
\hline Tabagistas"**** & 179 & 56,7 & 188 & \multirow{2}{*}{\multicolumn{2}{|c|}{$\begin{array}{c}35,14 \\
0,3\end{array}$}} & $3,16-7,35$ \\
\hline Sem informaçăo & & & 2 & & & \\
\hline
\end{tabular}

Total $316 \quad 100,0536100,0$

* O intervalo é estatisticamente significante ao nível de $\mathrm{p}<0,05$ se exclui 0 valor 1 .

* Nunca fumaram ou fumaram menos de 50 cigarros durantes toda a vida.

*** Categoria de referência.

**** Não fumavam ou abandonaram o hábito de fumar há 1 ou mais anos da data da entrevista.

***** Pessoas que fumavam ou que abandonaram o hábito de fumar há menos de 1 ano da data da entrevista.

Teste de tendência $59,47(p<0,001)$. fumado ou fumado menos de 50 cigarros durante toda a vida. Na Tabela 3 observa-se maior risco para o câncer de pulmão para os ex-fumantes em relaçāo aos não-fumantes e dos fumantes em relaçāo aos ex-fumantes. A quantidade de cigarros fumados, expressa em maços/ano, também mostra associação com a neoplasia pulmonar mantendo-se a tendência dose-resposta quando o grupo dos tabagistas foi analisado separadamente em relação aos não-tabagistas (Tabela 4).

A referência de outros cânceres na família mostra associação com a neoplasia pulmonar, considerando-se a referência a qualquer câncer e, especificamente, ao câncer de pulmảo, embora o teste de tendência não tenha sido estatisticamente significativo (Tabela 5).

$O$ efeito isolado da varíavel trabalho não apresentou resultados significativos (Tabela 2). $\mathrm{O}$ critério utilizado para definir trabalho industrial nesta fase de análise não permitiu separar adequadamente os expostos dos não-expostos. Procurou-se, então, categorizar a variável trabalho através da profissāo declarada pelo entrevistado. Definiram-se três grandes grupos para classificar os profissóes: (a) 
Tabela 4 - Distribuição de casos a controles, segundo a variável quantidade de cigarros fumados pelos tabagistas comparados com os năo-tabagistas $\theta$ odds ratio (OR) correspondente.

\begin{tabular}{|c|c|c|c|c|c|c|}
\hline \multirow[t]{2}{*}{ Maços/ano* } & \multicolumn{2}{|c|}{ Casos } & \multicolumn{2}{|c|}{ Controles } & \multirow[t]{2}{*}{ OR } & \multirow{2}{*}{$\begin{array}{c}\text { Intervalo } \\
\text { confiança } \\
95 \%^{* *}\end{array}$} \\
\hline & $N^{2}$ & $\%$ & $N^{2}$ & $\%$ & & \\
\hline Nenhum & 38 & 17,5 & 194 & 50,5 & $1,00^{* * * * *}$ & \\
\hline$>0$ a 20 & 23 & 10,6 & 47 & 12,2 & 2,50 & $1,30 \cdot 5,02$ \\
\hline$>20$ a 40 & 42 & 19,4 & 52 & 13,5 & 4,12 & $2,25-7,01$ \\
\hline$>40$ & 113 & 52,1 & 87 & 22,7 & $6,63^{*+* * *}$ & $4,06-10,38$ \\
\hline Sem informaçăo & 1 & 0,4 & 4 & 1,1 & & \\
\hline
\end{tabular}

Total

$2.17100,0384100,0$

* Número de maços de cigarros que uma pessoa fuma ou fumou por dia multiplicado pelo número de anos de hábito de fumar.

** $\quad 0$ intervaio é estatisticamente significante ao nivel de $p<0,05$ se exclui o valor 1 .

*** Não-tabagistas.

*** Categoria de referência.

***** Teste de tendencia $75,00(p<0,001)$.

Tabela 5 - Distribulção de casos e controles, segundo as variáveis outros cânceres e câncer de pulmăo na família comparadas com a nāo-referência a tumores malignos na famillia e adds ratio (OR) correspondente.

\begin{tabular}{|c|c|c|c|c|c|c|}
\hline \multirow{2}{*}{$\begin{array}{l}\text { Câncer na } \\
\text { família }\end{array}$} & \multicolumn{2}{|c|}{ Casos } & \multicolumn{2}{|c|}{ Controles } & \multirow[t]{2}{*}{ OR } & \multirow{2}{*}{$\begin{array}{c}\text { Intervalo } \\
\text { confiança } \\
95 \%\end{array}$} \\
\hline & № & $\%$ & $\mathrm{~N}^{\mathbf{2}}$ & $\%$ & & \\
\hline $\begin{array}{l}\text { Sem cáncer } \\
\text { Qualquer câncer }\end{array}$ & 260 & 82,3 & 461 & 86,0 & $1,00^{* *}$ & \\
\hline (exceto pulmão) & 47 & 14,9 & 70 & 13,1 & 1,19 & $0,78-1,81$ \\
\hline Cancer de pulmào & 9 & 2,8 & 5 & 0,9 & $3,20^{* k *}$ & $0,97-11,08$ \\
\hline
\end{tabular}

Total $316 \quad 100,0536100,0$

- O intervalo é estatisticamente significante ao nivel de $p<0,05$ se exclui o valor 1 .

** Categoria de referência.

*** Teste de tendência $3,35(p=0,067)$.

profissionais, abrangendo os profissionais liberais e executivos; (b) operários, comportando os trabalhadores ligados ao processo de produçāo industrial
Tabele 6 - Distribuiçāo de casos $\theta$ controles, segundo a variável profissão, categorizada por grupamentos profissionals*, e odds ratio (OA) correspondente.

\begin{tabular}{|c|c|c|c|c|c|c|c|}
\hline \multirow{2}{*}{\multicolumn{2}{|c|}{$\begin{array}{l}\text { Grupamento } \\
\text { profissional* }^{*}\end{array}$}} & \multicolumn{2}{|c|}{ Casos } & \multicolumn{2}{|c|}{ Controles } & \multirow[t]{2}{*}{ OR } & \multirow{2}{*}{$\begin{array}{l}\text { Intervalo } \\
\text { confiança } \\
95 \% \%^{* *}\end{array}$} \\
\hline & & $N^{2}$ & $\%$ & $\mathrm{~N}^{\mathrm{P}}$ & $\%$ & & \\
\hline $\begin{array}{l}\text { Profise } \\
\text { Outras } \\
\text { Operá } \\
\text { Sem ir }\end{array}$ & $\begin{array}{l}\text { sionals } \\
\text { ocupaçōes } \\
\text { irios*** } \\
\text { nformaçāo }\end{array}$ & $\begin{array}{r}11 \\
178 \\
109 \\
18\end{array}$ & $\begin{array}{r}3,5 \\
56,3 \\
34,5 \\
5,7\end{array}$ & $\begin{array}{r}13 \\
310 \\
186 \\
27\end{array}$ & $\begin{array}{r}2,4 \\
57,8 \\
34,7 \\
5,1\end{array}$ & $\begin{array}{l}1,00^{* * *} \\
1,47 \\
1,44\end{array}$ & $\begin{array}{l}0,60-3,59 \\
0,58 \cdot 3,58\end{array}$ \\
\hline Total & & \multicolumn{2}{|c|}{$346 \quad 100,0$} & \multicolumn{2}{|c|}{536100,0} & & \\
\hline$* * * *$ & \multicolumn{7}{|c|}{$\begin{array}{l}\text { A categorizaçāo por grupamento profissional foi } \\
\text { feita a partir da profissāo declarada pelo paciente. } \\
\text { O intervalo é estatisticamente significante ao nivel } \\
\text { de } p<0,05 \text { se exclui o valor } 1 \text {. } \\
\text { Catecoria de referência. }\end{array}$} \\
\hline
\end{tabular}

e (c) outras profissões, composto pelas profissões não incluídas nas categorias anteriores. As associações encontradas não foram estatisticamente significativas (Tabela 6). Estes resultados refletem o baixo poder discriminante conferido pela profissão declarada pelo entrevistado para classificar indivíduos expostos e não-expostos a cancerígenos.

\section{Análise Estratificada}

A partir da "Classificação de Exposiçāo Ocupacional a Cancerígenos por Escores" e com a variação dos escores na escala logarítmica, foram definidos quatro aglomerados, pressupondo-se quatro diferentes níveis de exposição ocupacional para a neoplasia pulmonar. O número dos casos e controles em cada aglomerado é apresentado na Tabela 7. Observa-se uma relação dose-resposta crescente do primeiro ao quarto aglomerado, com teste de tendência significante estatisticamente. Quando comparado o aglomerado 4 com o 1, o OR: 2,54 foi significativo ( $95 \%$ IC: 1,56 a 4,16 ).

Tabela 7 - Distribuiçăo de casos e controles em aglomerados por faixa de escore na escala logarítmica, segundo a Classificaçāo de Exposição Ocupacional a Cancerígenos por Escores e odds ratio (OR) correspondente.

\begin{tabular}{|c|c|c|c|c|c|c|c|}
\hline \multirow[t]{2}{*}{ Aglomerados } & \multirow{2}{*}{$\begin{array}{l}\text { Variação do } \\
\text { escore na escala } \\
\text { logarítmica }\end{array}$} & \multicolumn{2}{|c|}{ Casos } & \multicolumn{2}{|c|}{ Controles } & \multirow[t]{2}{*}{ OR } & \multirow{2}{*}{$\begin{array}{c}\text { Intervalo } \\
\text { confiança } \\
95 \% *\end{array}$} \\
\hline & & $\mathrm{N}^{2}$ & $\%$ & $\mathrm{~N}^{2}$ & $\%$ & & \\
\hline $\begin{array}{l}1 \\
2 \\
3 \\
4\end{array}$ & $\begin{array}{r}0 \text { a } 4,00 \\
4,02 \text { a } 4,92 \\
4,93 \text { a } 5,83 \\
5,84 \text { a } 7,96\end{array}$ & $\begin{array}{l}95 \\
86 \\
84 \\
51\end{array}$ & $\begin{array}{l}30,1 \\
27,2 \\
26,6 \\
16,1\end{array}$ & $\begin{array}{r}218 \\
142 \\
130 \\
46\end{array}$ & $\begin{array}{r}40,7 \\
26,5 \\
24,3 \\
8,6\end{array}$ & $\begin{array}{l}1,00^{* *} \\
1,39 \\
1,48 \\
2,54^{* * *}\end{array}$ & $\begin{array}{l}0,95 \cdot 2,03 \\
1,01-2,17 \\
1,56-4,16\end{array}$ \\
\hline Total & & 316 & 100,0 & 536 & 100,0 & & \\
\hline
\end{tabular}

* O intervalo é estatisticamente significante ao nivel de $p<0,05$ se exclui o valor 1 .

** Categoria de referência.

*** Teste de tendéncia $14,46(p<0,001)$. 
Na Tabela 7, a análise não contempla o controle de possíveis variáveis de confusão.

Para examinar o efeito conjunto das variáveis sob estudo, explorando as variáveis que pudessem estar interferindo na relação principal estudada, foram controladas as variáveis idade e sexo e, ainda, outras duas, que mostraram OR elevados quando analisadas isoladamente: tabagismo e câncer de pul-

Tabela 8 - Odds ratio (OR) e respectivos intervalos de confiança da análise estratificada da exposição ocupacional, controlando as variáveis tabagismo, câncer de pulmāo na famllia, sexo e idade.

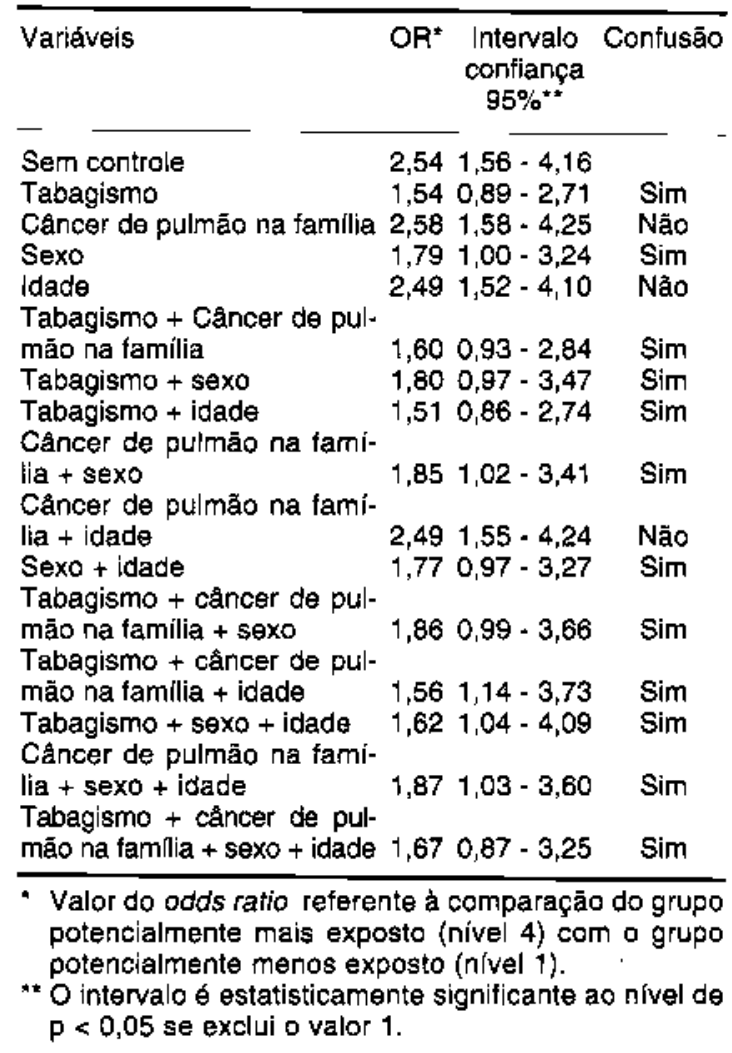

mão na família. Procedeu-se então à análise estratificada passo a passo, considerando-se inicialmente o controle das variáveis uma a uma, posteriormente duas a duas, a seguir três a três e, finalmente, as quatro variáveis simultaneamente (Tabela 8).

Como se pode observar, as variáveis câncer de pulmão na família e idade não indicaram estar confundindo os resultados, pois mostraram OR (quando comparado o último conglomerado com o de referência) muito próximo do identificado na análise sem controle. Também o controle conjunto destas duas variáveis nāo mostrou confusão. $O$ procedimento indicou como variáveis potencialmente confundidoras tabagismo e sexo, que sempre apresentaram tendência a modificar o OR quando presentes, tanto quando tratadas isoladamente quanto em conjunto com outras variáveis.

Na Tabela 9 é apresentada a análise estratificada para os quatro aglomerados de escores com o controle conjunto das variáveis: tagabismo, câncer de pulmão na família, sexo e idade. Os resultados indicam tendência crescente do efeito dose-resposta.

\section{Análise de Regressão Logística}

Sendo a neoplasia pulmonar a variável dependente, representada através da condição dicotômica: caso=1 (presença de câncer de pulmão) ou controle $=0$ (ausência de câncer de pulmão), a regressão logística estima o efeito de uma determinada variável sobre a ocorrência da neoplasia pulmonar na presença de outras variáveis independentes.

A análise de regressão logística não-condicional contemplou todas as variáveis simultaneamamente. O resultado para cada variâvel, considerando-se o coeficiente (estimativa do parâmetro multivariado), o erro padrão e a significância estatística, está na Tabela 10.

Tabela 9 - Distribuiçāo de casos e controles em aglomerados por faixa de escore na escala logaritmica, segundo a Classificaçăo de Exposiçăo Ocupacional a Cancerígenos por Escores, com controle das variáveis sexo, idade, tabagismo e cancer de pulmăo na familia, e odds ratio (OR) correspondente.

\begin{tabular}{|c|c|c|c|c|c|c|c|}
\hline \multirow[t]{2}{*}{ Aglomerados } & \multirow{2}{*}{$\begin{array}{l}\text { Variaçāo do } \\
\text { escore na escala } \\
\text { logarítmica }\end{array}$} & \multicolumn{2}{|c|}{ Casos* } & \multicolumn{2}{|c|}{ Controles* } & \multirow[t]{2}{*}{ OR } & \multirow{2}{*}{$\begin{array}{c}\text { Intervalo } \\
\text { confiança } \\
95 \% * *\end{array}$} \\
\hline & & $N^{\circ}$ & $\%$ & $N^{2}$ & $\%$ & & \\
\hline$T$ & $0=4,00$ & 94 & 29,9 & 217 & 40,7 & $1,00^{\star \star *}$ & \\
\hline 2 & 4,02 a 4,92 & 86 & 27,4 & 142 & 26,6 & 1,09 & $0,68-1,72$ \\
\hline 3 & 4,93 a 5,83 & 83 & 26,4 & 130 & 24,4 & 1,26 & $0,74-2,15$ \\
\hline \multicolumn{7}{|c|}{5,84 \& 7,20} & \\
\hline Total & & 314 & 100,0 & 533 & 100,0 & & \\
\hline
\end{tabular}

* 2 casos e 3 controles perdidos por falta de informaçōes sobre variáveis.

** $O$ intervalo é estatisticamente significante ao nivel de $p<0,05$ se exclui 0 valor 1

*** Categoria de referência. 
Tabela 10 - Coeficiente específico, erro padräo e nivel de significancia obtido pela análise de regressão logística nāo-condicional para as variáveis exposição ocupacional*, sexo, idade, tabagismo e cáncer de pulmâo na familia.

\begin{tabular}{lccc}
\hline Variáveis & Coeficiente Erro padrão & $p^{* *}$ \\
\cline { 4 - 5 } Exposição ocupacional & 0,2262 & 0,086 & 0,008 \\
Sexo & 0,2257 & 0,209 & 0,280 \\
Idade & 0,0190 & 0,008 & 0,013 \\
Tabagismo & 0,0179 & 0,002 & $<0,001$ \\
Câncer pulmăo família & 0,2701 & 0,177 & 0,127 \\
\hline
\end{tabular}

* Classificação de Exposiçäo Ocupacional a Cancerlgenos por escores, grupos de escore aglomerados pelas diferenças entre as medias na escala logarítmica.

** Nivel de significancia.

O coefíciente obtido para a exposição ocupacional significa um aumento da probabilidade de câncer de pulmão da ordem de $e^{0,2262}$ para cada diferente nível de exposição.

Como está sendo usada a escala logarítmica:

Ln $(\mathrm{OR})=0,2262$ (diferença dos níveis de exposição)

$\mathrm{OR}=\mathrm{e}^{0.2262}$

Foram definidos quatro níveis de exposiçāo (quatro aglomerados). $\mathrm{O}$ aglomerado 1 é a categoria de referência. Entāo, para avaliar o último nível de referência (aglomerado 4 - comportando aqueles com maior probabilidade de exposição a cancerígenos) em relação à categoria de referência (aglomerado 1 - composto por aqueles com menor probabilidade de exposição a cancerígenos), temos:

Log ${ }^{\text {(odds) }}$ Expostos $=0,2262 \times 4$

Log ${ }^{\text {(odds) } N a ̂ 0-E x p o s t o s ~}=0,2262 \times 1$

Log (odss Expostos/odds Não-Expostos) = Log ${ }^{(\text {dods })}$ Expostos - Log ${ }^{\text {(odds) }}$ Não- Expostos $=0,2262(4-1)$ $\log (\mathrm{OR})-0,2262(3)$

$\mathrm{OR}=\mathrm{e}^{0.2262 \times 3}$

Obteve-se, então, um OR estatisticamente significante de 1,97 (95\% IC: 1,52 a 2,55). Este resultado é semelhante ao detectado pela análise estratificada.

\section{Discussăo}

A análise do efeito isolado das variáveis sob estudo identificou prontamente o tabagismo como fator associado ao câncer de pulmão (Tabela 1). A consistência desta associação aumentou na medida em que os procedimentos de análise buscaram definir uma relação dose-resposta (Tabelas 2,3 e 4). Esta associação detectada na RMSP apenas confirma as identificadas em outros estudos realizados nas últimas quatro décadas em países desenvolvidos ${ }^{4.13 .22 .23}$. O grau da associação encontrado para os fumantes de mais de 40 maços/ano OR: 6,63 (95\% IC: 4,06 a 10,38), entretanto, foi menor que o identificado em outros estudos ${ }^{i 1}$. Isto pode ser explicado pelo fato do efeito tabagismo, no presente estudo, apresentar distorçāo. Nāo foi tomada nenhuma precaução especial com relação a possíveis vieses de seleção, ligados ao tabagismo, na escolha dos controles, pois não era objetivo principal o exame desta varível sobre a neoplasia pulmonar. Note-se que muitos controles tinham patologias relacionadas a este fator de risco. Assim, a estimativa do efeito do tabagismo sobre a incidência de câncer de pulmão está subestimada.

A variável câncer de pulmão em familiares mostrou OR alto: 3,20. O resultado nāo foi significativo estatisticamente. Entretanto, o limite inferior do intervalo de confiança (95\% IC: 0,97 a $11,08)$, muito próximo de 1 , indica tendência no sentido de associação válida e que poderia ser identificada com uma amostra maior.A análise do efeito isolado do fator trabalho identificou uma fraca associação entre trabalho industrial e carcinoma broncogênico (Tabela 2). Também o estudo por categorias profissionais nāo mostrou correlaçōes estatisticamente significativas com a doença (Tabela 6). Foi fundamental definir critérios de exposição com maior clareza para separar, de forma mais eficiente, os expostos dos não-expostos e, assim, permitir avaliar a contribuição dos riscos ocupacionais na determinação do carcinoma broncogênico.

A alternativa de conferir escores individuais segundo a ocupaçāo, setor de trabalho, exposição a substâncias químicas e o tempo de trabalho, pressupõe que os maiores escores correspondem às pessoas com maiores probalbilidades de terem sido expostas a agentes cancerígenos no trabalho por tempo mais prolongado. Nesse sentido, se a variação dos escores atribuídos oscilou entre 0 (zero) e 1.732 , por hipótese, aquele indivíduo com escore individual final de 1.732 teve maior probabilidade de exposição a substâncias cancerígenas na vida profissional do que aquele com escore zero.

$\mathrm{Na}$ análise estratificada, sem o controle de variáveis confundidoras, observou-se OR de 2,54, estatisticamente significativo, quando comparado o grupo de maiores escores com aquele detentor de escores mais baixos (Tabela 7). Na análise estra- 
tificada, com controle das variáveis sexo, idade, tabagismo e câncer de pulmão na família, embora tenha se mantido o efeito dose-resposta, os OR não foram significativos estatisticamente (Tabela 9).

Os grandes intervalos de confiança encontrados na análise estratificada com controle podem ser imputados ao grande número de estratos criados pela combinação dos diferentes níveis dessas variáveis: sexo (duas categorias); idade (cinco categorias); tabagismo (três categorias); câncer de pulmão na família (três categorias). Têm-se, então, 90 estratos e, em cada estrato, quatro categorias de exposição ocupacional. Com este grande número de estratos e consequiente pequeno número de observaçōes em cada um deles, a variabilidade das estimativas, expressa pelos intervalos de confiança, tornou-se maior. Saliente-se que dos 90 estratos possíveis, a análise restringiu-se a apenas 60 , devido à ausência de observaçōes nos outros 30 estratos. Esta é uma limitação da análise estratificada.

A limitação decorre da ampla dispersão dos dados quando há grande número de variáveis ou pelo emparelhamento por muitas categorias em cada variável, pois isto aumenta a perda de informações levando, portanto, a estimativas pouco precisas.

A regressão logística não-condicional revelou um OR estatisticamente significante de 1,97 quando indivíduos com maior probabilidade de risco de exposição a susbstâncias cancerígenas (maiores escores) foram comparados àqueles com menor risco (menores escores). Assim, assumindo-se o dado do Registro de Câncer de São Paulo $^{18}$ de 36,5/100,000 habitantes como base da incidência de câncer de pulmão na RMSP, este OR de 1,97 indica que a incidência de neoplasia pulmonar entre a população com maior risco de exposição a cancerígenos (com características semelhantes àquela do aglomerado 4) seria de $71,9 / 100.000$ habitantes.

Os resultados do presente estudo revelam semelhanças com outros estudos caso-controle realizados para estudar a associação dos riscos ocupacionais ao câncer de pulmão em países desenvolvidos. É importante sublinhar, entretanto, certas diferenças. Estudos com a estratégia aqui desenvolvida de rastrear de forma indiscriminada riscos ocupacionais a que estiveram expostos casos e controles, nāo são comuns. Jöckel e col. ${ }^{10.11}$ relataram os resultados de um estudo caso-controle na Alemanha: os OR só se tornaram expressivos e estatisticamente significativos na medida em que se analisaram ramos específicos de atividade. Outros estudos têm mostrado OR similares aos obtidos aqui e, mesmo, menores e nāo significativos ${ }^{1,6.1 \% 19}$.

Outro aspecto a ser destacado diz respeito à classificação desenvolvida para avaliar a exposição ocupacional. É possível que este critério de escores possa ser melhorado ou mesmo totalmente repensado. De qualquer forma, a classificação permitiu explorar as exposiçōes ocupacionais nas suas relações cotn a neoplasia pulmonar.

Tendo no horizonte tais limitaçōes e restriçōes, os resultados obtidos permitem assumir que trabalhadores que estiveram ligados aos setores de produção de ramos de atividade industriais, nos quais havia maior probabilidade de exposição a substâncias cancerígenas, têm cerca de duas vezes o risco de virem a desenvolver carcinoma broncogênico quando comparados a trabalhadores de outros setores de atividade onde, provavelmente, a exposição a cancerígenos é menor.

Concluj-se, portanto, que o câncer de pulmão, de modo análogo a outras doenças, não se distribui homogeneamente na populaçāo trabalhadora. A lógica que rege a inserção no mercado de trabalho submete os indivíduos a riscos diferenciados para a neoplasia pulmonar.

\section{Agradecimentos}

Às equipes médicas e de enfermagem dos hospitais onde o trabalho foi realizado, particularmente aos clínicos a seguir indicados, pela imprescindível cooperação na realização da pesquisa:

Dr. Antonio Pedro Mirra; Dr. José Rodrigues Pereira; Dr. João Valente Barbas Filho; Dra. Tereza Takagaki; Dr. Hakaru Tadakaro; Dr. Sérgio Jamnik; Dr.Salvador Mercúrio; Dr. Jorge Afiune; Dr. Nelson Morrone; Dr. Luiz Augusto Marcondes Fonseca; Dra. Ilka Lopes Santoro; Dr. Mozart Tavares de Lima; Dr. Nelson Morrone; Dr. Carlos Alberto de Castro Pereira; Dr. Jorge Nakateni; Dr. Roberto Saad Júnior; Dr. Jorge Ethel Filho. 


\section{Referênclas Bibliográficas}

1. BOND, G.G. et al, Nested case-control study of lung cancer among chemical workers. $A m . J$. Epidemiol., 124: 53-66, 1986.

2. BRESLOW, N.E. \& DAY, N.E. Statistical methods in cancer researcb: the analysis of case-control studies. Lyon, International Agency for Research on Cancer, 1980. (LARC-Scientific publications, 32), v. 1

3. CENTERS FOR DISEASE CONTROL AND PREVENTION. Trends in lung cancer incidence and mortality - United States, 1980 - 1987. MMWR, 39: 875, 881-3, 1990.

4. DOLJ, R. \& HILL, A.B. Smoking and carcinoma of the lung: preliminary report. Brit. Med.J., 2: 739-48, 1950.

5. DOLL, R. \& PETO, R. The causes of cancer: quantitative estimates of avoidable risks of cancer in the United States today. New York, Oxford University Press, 1981.

6. GUSTAVSSON, P. et al. Lung cancer and exposure to diesel exhaust among bus garage workers. Scand. J. Work Envinon. Health, 16: 348-54, 1990 .

7. HOWE, G.M. International variations in cancer incidence and mortality. In: Howe, G.M. Global cancerology: a world geograpby of cancers. Edinburgh, Churchill Livinstone, 1986. p. 3-42.

8. INTERNATIONAL LABOUR OFFICE. Occupational cancer: prevention and control. Geneva, 1988. (Occupational Safety and Health Series, 39).

9. JOHNSON, R.A. \& WICHERN, D.W. Applied multivariate statistics analysis. Englewood Cliffs, Prentice Hall, 1982.

10. JÖCKEI, K. H. et al. Lung cancer risk and welding - preliminary results from an ongoing case-control study. Am. J. Industr. Med., 25: 805-12, 1994.

11. JÖCKEL, K.H. et al. Occupational and environmental hazardz associated with lung cancer. Int. J. Epidemiol., 21: 202-13, 1992.
12. KELSEY, J.L. et al. Metbods in obsenational epidemiology. New York, Oxford University Press, 1986.

13. LEVIN, M.L. et al, Cancer and tobacco smoking: preliminary report. JAMA, 143: 336-8, 1950.

14. MANTEL, N. \& HAENSZEL, W. Statistical aspects of the analysis of data from retrospective studies of disease. J. Nat. Cancer Inst., 22: 719-48, 1959.

15. MEIJERS, J.M.M. et al. Silica exposure and lung cancer in ceramic workers: a case-control study. Int. J. Epidemiol., 19: 19-25, 1990.

16. MENDONÇA, G.A.S. Câncer no Brasil: um risco crescente. Rev. Bras. Cancerol, 38: 167-76, 1992.

17. MINISTÉRIO DA SAÚDE. Secretaria Nacional de Açòes Básicas de Saúde. Subsistema de Informaçōes sobre Mortalidade. Estatística de montalidade, Brasil, 1988. Brasília, Centro de Documentação do Ministério da Saúde, 1993.

18. MIRRA, A.P. \& FRANCO, E.L. Incidencia do câncer no Municipio de Säo Paulo, Brasil: $1969,1973,1978$. São Paulo, Registro de Câncer de São Paulo/Instituto Ludwig para Pesquisa de Câncer, 1985.

19. RINSKY, R.A et al. Case-control study of lung cancer in civilian employees at the Portsmouth Naval Shipyard, Kittery, Maine. Am.J. Epidemiol, 127: 55-64, 1988.

20. SCHOTTENFELD, D. \& FRAUMENI JR, J.F. Cancer epidemiology and prevention. Philadelphia, W.S. Saunders Company, 1982.

21. WATHERHOUSE, J. et al. Cancer incidence in five continents. Lyon, International Agency for Research on Cancer, 1982. (IARC Scientific Publication, 42).

22. WYNDER, E.L. \& GOODMAN, M. T. Smoking and lung cancer: some unresolved issues. Epidemiol. Rev., 5: 177-207, 1983.

23. WYNDER, E.L. \& GRAHAN, E.A. Tobacco smoking as a possible etiological factor in bronchiogenic carcinoma. JAMA, 143: 329-36, 1950.

\begin{abstract}
In a hospital-based case-control study, 316 lung cancer cases and 536 controls were interviewed for their occupetional, smoking, passive smoking, cancer in the family and residential histories as well as social economic status, by trained interviewers, using a standardized questionneire. Cases and controls were matched by hospital, sex and age. The study was carried out between 1st July 1990 and 31 st January 1991 in 14 hospitals in the Metropolltan Region of S. Paulo, the most highly industrialized and urbanized region in Brazil. Score criteria were developed for the ordering of the individuals of the study by occupational exposure to know carcinogens to the lung. in order to evaluate this exposure during the occupational life of each person. The criteria accumulated information on exposure to carcinogens as regards type, sector of work and time in each employment. The unconditional logistic regression analysis showed an odds ratio of 1.97 (95\% IC: 1.52 to 2.55) for the highest exposure group. This result showed that workers linked to the production sectors of several industries have about twice the risk of developing lung cancer as workers involved in non-industrial activities.
\end{abstract}

Lung neoplasms, epidemiology. Occupational exposure, adverse effects. Industry. 